\title{
Pendlermobilität in der Nordwestschweiz Entwicklung der Pendlerbeziehungen und der Verkehrsmittelwahl zwischen 1970 und 1990
}

\author{
Patrick Rey, Basel
}

\section{Einleitung}

Die Pendlermobilität in der Schweiz hat sich zwischen 1970 und 1990 in vielfacher Hinsicht verändert. Die Zahl der erwerbstätigen Pendler erhöhte sich um $30 \%$. Der Anteil des motorisierten Privatverkehrs stieg von $38 \%$ auf $52 \%$, womit das Auto zum wichtigsten Verkehrsmittel wurde. Gleichzeitig sank der Anteil der zu Fuss pendelnden Erwerbstätigen von 26\% auf 10\% (Bundesamt Für STatISTIK BFS 1990: 11). Die diesem Artikel zu Grunde liegende Untersuchung hatte zum Ziel, die Entwicklung der regionalen Pendlermobilität der Nordwestschweiz im Kontext des sich verändernden Mobilitätsverhaltens und der funktionalen Entmischung zu untersuchen.

\subsection{Pendlermobilität als Ursache und Auswirkung funktionaler Entflechtung}

Pendler sind Erwerbstätige, welche eine räumliche Distanz zwischen Wohn- und Arbeitsort zurücklegen. Pendlermobilitäts-Kenngrössen sind folglich bedeutsam als Indikatoren für Suburbanisierungsprozesse. Es kommt hinzu, dass der Pendlerverkehr zwischen Wohn- und Arbeitsorten nicht nur die Auswirkungen der funktionalen Entmischung abbildet, sondern selbst zu einer der Hauptursachen wurde. Nach FrEY (1996: 13-15) sind zwei sich selbst verstärkende Kreisläufe wichtig:

- Durch den Ausbau der Verkehrsinfrastruktur sinken die Raumüberwindungskosten. Schnellere Verbindungen senken die Zeitkosten und erhöhen die Attraktivität peripherer Wohnorte. Dieser und weitere Pull-Effekte, wie z.B. der Wunsch nach dem Leben im Grünen oder einer kindgerechten Umgebung, werden durch kernstädtische Push-Effekte noch verstärkt. Neuer Pendlerverkehr wird aber erst dadurch induziert, dass oft nur der Wohnort in die Peripherie verlegt werden kann. Der Arbeitsplatz bleibt in der Regel in der Zentrumsgemeinde.

- Die Verkehrszunahme führt vor allem in den Kernstädten und entlang von Hauptverkehrsachsen zu sinkender Umweltqualität und einer Verschlechterung des Wohnumfeldes, so dass durch die Umweltbeeinträchtigungen Sub- und Desurbanisationsprozesse selbstverstärkend beeinflusst werden.

\subsection{Pendlerverkehr und externe Umwelt- und Gesundheitskosten}

Eine Partialanalyse über die Kosten der Luftverschmutzung in den beiden Basel ergab monetarisierbare, durch den Verkehr verursachte externe Kosten durch die Luftverschmutzung im Rahmen von CHF 210-270 Mio. pro Jahr, wobei die effektiven externen Kosten noch höher liegen, da eine Reihe weiterer externer Kosten (Lärm, Unfallfolgekosten, anthropogen verursachter Treibhauseffekt usw.) nicht berücksichtigt wurde (ECONCEPT 1998: 1-4). Im Kontext der Diskussionen über die durch den Verkehr verursachten externen Kosten stellt daher das zeitliche Monitoring der Entwicklung der Pendlermobilität auch eine wichtige Grundlagenarbeit für das Umweltmonitoring in der Region Nordwestschweiz dar.

\section{Grundlagen der Untersuchung}

Funktionale Entmischungsprozesse liessen vermuten, dass sich die räumliche Trennung von Wohnen und Arbeiten auch in der Nordwestschweiz zwischen 1970 und 1990 weiter akzentuierte. Eine umfassende empirische Überprüfung stand aber noch weitgehend aus. Dasselbe galt für Veränderungen in der Verkehrsmittelwahl. Aus den skizzierten Entwicklungsprozessen wurden folgende Arbeitshypothesen abgeleitet:

- Die interkommunale Pendlermobilität nahm aufgrund der sich akzentuierenden räumlichen Trennung von Wohnen und Arbeiten in der ganzen Nordwestschweiz zwischen 1970 und $1990 \mathrm{zu}$. Äquivalent dazu ist davon auszugehen, dass der Anteil der in der Wohngemeinde arbeitenden Erwerbstätigen im Untersuchungszeitraum abnahm, wobei vor allem periphere ländliche Gemeinden eine überdurchschnittliche Abnahme verzeichneten.

- Die Pendlermobilität wurde trotz einem starken Wachstum im peripheren, ländlichen Raum auch im Jahr 1990 durch Pendlerströme innerhalb und in die Agglomeration dominiert.

- Der öffentliche Verkehr konnte in der Nordwestschweiz seinen Anteil in den 80er Jahren steigern. Die anhaltende Besiedlung peripherer Regionen der Nordwestschweiz führte aber auch dazu, dass der motorisierte Individualverkehr ausserhalb der Agglomeration Basel stärker gewachsen ist als der öffentliche Verkehr. 


\subsection{Räumliche Abgrenzung der Nordwestschweiz}

Die Fokussierung auf die räumliche Analyse der sich im Zeitverlauf ändernden Pendlerbeziehungen bedingt die Festlegung räumlicher Grenzen. Die Region Nordwestschweiz setzt sich zusammen aus den beiden Kantonen Basel-Stadt (Stadt Basel, Riehen und Bettingen) und Basel-Landschaft (Bezirke Arlesheim, Laufen, Liestal, Sissach und Waldenburg), den solothurnischen Bezirken Dorneck und Thierstein sowie den aargauischen Bezirken Rheinfelden, Laufenburg und einigen Gemeinden des Bezirks Brugg. Die Nordwestschweiz wird naturräumlich geprägt durch den Tafeljura und die südlich anschliessenden Gebirgszüge des Faltenjuras. Die bewaldeten, ländlichen und landwirtschaftlich genutzten Tafeljura-Hochflächen und die dicht besiedelten Täler der sie von Norden zur südlichen Tiefenlinie des Rheins durchschneidenden Flüsse von Birs, Ergolz und Sissle charakterisieren das Innere der Region Nordwestschweiz. Südlich der Jurahöhen grenzt die Nordwestschweiz an das Schweizer Mittelland. Obwohl es sich bei der Nordwestschweiz nicht um eine politische Region handelt, stellt die gewählte Abgrenzung Nordwestschweiz nicht ein wissenschaftliches Konstrukt dar, das sich nur zu dem hier interessierenden Zweck besonders gut eignen würde. Vielmehr wird die Nordwestschweiz trotz der Gebietszugehörigkeit zu vier Kantonen nicht nur als die Summe der Kantonsteile wahrgenommen. Die Stadt Basel stellt mit ihrer wirtschaftlichen und kulturellen Zentrumsfunktion für die Agglomerationsgemeinden und peripheren Räume der Nordwestschweiz eine Kernstadt dar, deren Leistungsangebot von grosser regionaler Bedeutung ist.

\subsection{Datengrundlagen}

Grundlage für die Analyse des werktäglichen Pendelns zwischen Wohn- und Arbeitsort waren kommunale Daten der eidgenössischen Volkszählungen 1970, 1980 und 1990. Saisonale Pendler, Wochenpendler, Schüler, Studenten und im Ausland wohnhafte Personen (Grenzgänger) wurden aus methodischen und datentechnischen Gründen nicht in die Untersuchung miteinbezogen. Die Analysen konzentrierten sich im weiteren auf inter- und intrakommunale Pendlerbeziehungen innerhalb der Nordwestschweiz.

\subsection{Pendlermobilitäts-Kenngrössen}

Die empirischen Untersuchungen konzentrierten sich auf eine Zeitreihenanalyse der inter- und intrakommunalen Pendlermobilität, wobei die verschiedenen Facetten mittels charakteristischen Kenngrössen (Wegund Zupendleranteile, Pendlersaldo usw.) analysiert wurden.

- Interkommunale Pendlermobilität: Ein Schwerpunkt der empirischen Analyse war die Betrachtung der räumlichen Entwicklung der interkommunalen Pendlerströme. Interkommunale Pendler sind Er- werbstätige, deren Wohn- und Arbeitsgemeinden nicht identisch sind. Sie sind Wegpendler der Wohngemeinde $X$ und Zupendler der Arbeitsgemeinde $\mathrm{Y}$.

- Intrakommunale Pendlermobilität: Analog zur Betrachtung der interkommunalen Pendlerströme erfolgte eine Zeitreihenanalyse der intrakommunalen Pendler, das heisst derjenigen Pendler, deren Wohnund Arbeitsort innerhalb derselben Gemeinde liegt (Wohn- =Arbeitsgemeinde).

- Verkehrsmittelorientierte Betrachtungen: Neben verkehrsmittelunabhängigen Analysen der inter- und intrakommunalen Pendlermobilität stand die Entwicklung der Verkehrsmittelwahl im Zentrum. Dabei wurden nicht alle Verkehrsmittel einzeln betrachtet. Der «Öffentliche Verkehr» (ÖV) und der «Motorisierte Individualverkehr» (MIV) standen im Zentrum der Betrachtung. Der MIV setzt sich in dieser Untersuchung zusammen aus den Verkehrsmitteln Personenwagen (als Lenker und Mitfahrer) und Motorrad/Roller. Der ÖV wiederum fasst die Verkehrsmittel Eisenbahn, Tram/städtischer Bus/Trolleybus und Postauto/Überlandbus zusammen.

Die nachfolgende Darstellung der empirischen Ergebnisse orientiert sich im wesentlichen an der Unterscheidung zwischen inter- und intrakommunalem Pendlerverkehr.

\section{Arbeiten ausserhalb der Wohngemeinde: Von der Minderheit zur Mehrheit}

Unabhängig von der Verkehrsmittelwahl wurde die Entwicklung der interkommunalen Pendlermobilität zwischen 1970 und 1990 primär durch die zunehmende Trennung von Wohn- und Arbeitsort der innerhalb der Nordwestschweiz wohnenden Erwerbstätigen gesteuert und weniger durch das Wachstum der sich neu in der Nordwestschweiz niederlassenden Erwerbstätigen. Denn die Zahl der Erwerbstätigen nahm im Untersuchungszeitraum nur um rund 20'000 zu, während die Zahl der interkommunal pendelnden Erwerbstätigen um 58'800 Personen zunahm (Abb. 1). Diese Entwicklung lässt sich nur dadurch erklären, dass für immer mehr Erwerbstätige die Wohngemeinde nicht mehr identisch ist mit der Arbeitsgemeinde. Der Anteil der interkommunalen Pendler stieg zwischen 1970 und 1990 von $33 \%$ auf $52 \%$. Damit überschritt 1990 mehr als jeder zweite Erwerbstätige auf dem Arbeitsweg eine Gemeindegrenze, während 1970 erst jeder dritte Erwerbstätige ausserhalb der Wohngemeinde arbeitete.

\subsection{Die Stadt Basel bleibt dominant}

Im Jahr 1990 pendelten 45 von 100 interkommunalen Pendlern von ihrem Wohnort in die Stadt Basel zur Arbeit. Trotz einer geringen Abnahme des Anteils 




Abb. 1: Pendler in der Nordwestschweiz

Commuters in North-West Switzerland

Les pendulaires dans le nord-ouest de la Suisse

Quelle: BundeSAMT Für STATISTIK: Volkszählungen 1970, 1980, 1990, eigene Berechnungen

der nach Basel pendelnden Erwerbstätigen bleibt die Stadt Basel das dominierende Beschäftigungszentrum (Abb. 2). Wird die Veränderung der übrigen Agglomerationsgemeinden berücksichtigt, dann pendelten 1990 wie schon 1970 rund $75 \%$ aller interkommunalen Pendler in die Stadt Basel oder in eine der übrigen Agglomerationsgemeinden. Die Entwicklung der Pendlermobilität in der Agglomeration Basel ist damit auch ein mobilitätsspezifisches Indiz für die weiterhin hohe Arbeitsplatzkonzentration innerhalb der Agglomeration und die geringe Arbeitsplatzsuburbanisierung in die übrigen Gemeinden der Nordwestschweiz.

Bei der Betrachtung der Arbeitsorte der in den Agglomerationsgemeinden wohnhaften Pendler fiel auf, dass der Anteil der in Basel arbeitenden Pendler zwischen 1970 und 1990 von $73 \%$ auf $65 \%$ abgenommen hat, während der Anteil der zwischen Agglomerationsgemeinden (ohne Stadt Basel) pendelnden Erwerbstätigen von $20 \%$ auf $27 \%$ zugenommen hat. Obwohl auch 1990 noch rund zwei von drei in den Agglomerationsgemeinden wohnhaften Pendlern in der Stadt Basel arbeiteten, zeigt sich die zunehmende Bedeutung der übrigen Agglomerationsgemeinden als Arbeitsorte. Dies zeigt sich unter anderem auch in der Entwicklung der Beschäftigtenzahl. Während die Zahl

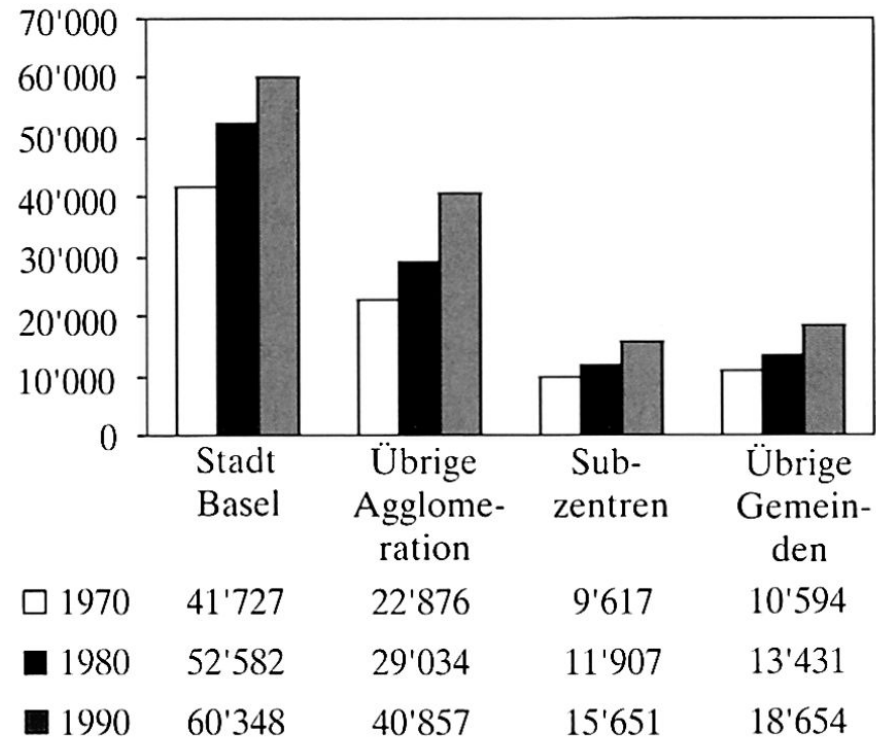

Abb. 2: Verteilung der Pendler nach Zielorten Distribution of commuters according to destination Distribution des pendulaires selon leur destination Quelle: Bundesamt Für STATISTIK: Volkszählungen 1970, 1980, 1990, eigene Berechnungen 
der in der Stadt Basel Beschäftigten zwischen 1975 und 1995 um rund 21'000 Personen zunahm, verzeichneten die (übrigen) Agglomerationsgemeinden ein Wachstum von rund $26^{\prime} 000$ Beschäftigten. Diese Beschäftigungsentwicklung ist ein starkes, die Ergebnisse der interkommunalen Pendlermobilität unterstützendes Indiz. Trotzdem bleibt die Stadt Basel das wirtschaftliche Zentrum der Region Nordwestschweiz. Dies zeigt sich nicht zuletzt daran, dass die Zahl der in den Agglomerationsgemeinden wohnenden und in Basel arbeitenden Erwerbstätigen auch zwischen 1980 und 1990 um weitere 7\% zugenommen hat. In der Entwicklung der interkommunalen Pendlermobilität zeigt sich, dass die Stadt Basel als Wohnort im Vergleich zu den übrigen Agglomerationsgemeinden weiter an Attraktivität verloren hat.

\subsection{Subzentren: Alternative Wohnstandorte für in der Agglomeration Beschäftigte}

Neben der Agglomeration Basel gibt es Gemeinden, welche auf regionaler Ebene eine Zentrumsfunktion für ländlich-periphere Umlandgemeinden erfüllen. Diese Gemeinden wurden als Subzentren bezeichnet und umfassen die Gemeinden Dornach, Laufen, Liestal, Sissach, Rheinfelden, Frick und Laufenburg.

Die zunehmende Bedeutung der regionalen Subzentren als Wohnorte für in anderen Gemeinden arbeitende Erwerbstätige zeigt sich darin, dass zwischen 1970 und 1990 der durchschnittliche Wegpendleranteil in den untersuchten Gemeinden von rund $29 \%$ auf $50 \%$ angestiegen ist. Bei der Betrachtung der Entwicklung der Zielorte der interkommunalen Pendler fällt auf, dass immer mehr in den Subzentren wohnhafte Pendler in der Agglomeration Basel arbeiten. Es zeigt sich, dass verkehrstechnisch gut an die Kernstadt und die übrigen Agglomerationsgemeinden angebundene Gemeinden vermehrt zum alternativen Wohnstandort für in der Stadt Basel arbeitende Erwerbstätige werden.

Auch in den übrigen Gemeinden der Nordwestschweiz ist analog zur Entwicklung in den regionalen Subzentren die Zahl der Wegpendler zwischen 1970 und 1990 stark angestiegen. Der prozentuale Wegpendleranteil erhöhte sich von durchschnittlich $47 \%$ auf $69 \%$. Zwei von drei Erwerbstätigen arbeiteten somit 1990 nicht in der Wohngemeinde. In der Agglomeration waren es drei von vier und in den Subzentren arbeitete jeder zweite Erwerbstätige ausserhalb der Wohngemeinde.

Fazit

Gesamthaft bestätigte sich die Hypothese, dass die interkommunale Pendlermobilität in der ganzen Nordwestschweiz zwischen 1970 und 1990 zugenommen hat, und dass trotz einer zunehmenden und sich in die Peripherie jenseits der Agglomeration Basel ausdehnenden Bevölkerungssuburbanisierung die interkommunale Pendlermobiliät durch den Pendlerverkehr innerhalb und in die Agglomeration Basel dominiert wird. Dies vor allem darum, weil sich das Arbeitsplatzangebot in der Agglomeration Basel konzentriert, so dass eine zunehmende Bedeutung peripherer Gemeinden als Wohnstandorte in eine Zunahme der interkommunalen Pendlermobilität mündet.

\subsection{In der Wohngemeinde arbeitende Erwerbstätige: Von der Mehrheit zur Minderheit}

Aufgrund der zunehmenden interkommunalen Pendlermobilität sank der Anteil der in der Wohngemeinde arbeitenden Erwerbstätigen zwischen 1970 und 1990 durchschnittlich von $65 \%$ auf $44 \%$. Trotz der flächendeckenden Abnahme (Karten 1 und 2) zeigen sich charakteristische Entwicklungsunterschiede. In der Stadt Basel betrug der prozentuale Anteil der in der Wohngemeinde arbeitenden Erwerbstätigen im Jahr 1990 trotz einer Abnahme von rund 10\% noch $81 \%$, während der Anteil in den übrigen Gemeinden der Nordwestschweiz zwischen 1970 und 1990 durchschnittlich von $51 \%$ auf $29 \%$ gesunken ist. In den regionalen Subzentren sank der Anteil durchschnittlich von $70 \%$ auf $47 \%$.

Der Vergleich der Entwicklungen zeigt, dass der Anteil um so grösser ist, je bedeutender eine Gemeinde als Lebens-, Kultur und Wirtschaftsstandort. Weitere Einflussfaktoren, welche die Faktoren «wirtschaftliche Zentralität» und «Urbanität» überlagern, sind die Grösse und Kernstadtnähe einer Gemeinde. Mit zunehmender Nähe nimmt der Charakter einer Gemeinde als alternativer Wohnort für in der Kernstadt arbeitende Erwerbstätige zu. So überrascht es wenig, dass die Agglomerationsgemeinden (ohne die Stadt Basel) 1990 mit durchschnittlich 24\% den tiefsten Anteil der in der Wohngemeinde arbeitenden Erwerbstätigen innerhalb der Nordwestschweiz aufwiesen.

\section{Verkehrsmittelwahl im interkommunalen Pendlerverkehr}

Der Anteil der Verkehrsmittel «Velo/Mofa», «Werkund Schulbus», «zu Fuss», und «andere» sank zwischen 1970 und 1990 durchschnittlich von $20 \%$ auf $9 \%$. Gleichzeitig stieg der durchschnittliche Anteil der mit dem ÖV und MIV pendelnden Erwerbstätigen von $80 \%$ auf $91 \%$. Die zusammenfassende Darstellung der Entwicklung der interkommunalen Verkehrsmittelwahl kann sich daher auf den öffentlichen Verkehr (ÖV) und den motorisierten Individualverkehr (MIV) beschränken. Bei der Betrachtung der Gesamtentwicklung ist einerseits zu unterscheiden zwischen ÖV und MIV und andererseits zwischen den Entwicklungen der 70er- und 80er Jahre.

Die 70er Jahre können im Vergleich zur Entwicklung in den 80er Jahren als Jahrzehnt der Automobilisierung bezeichnet werden. Trotz den Erdölkrisen der 


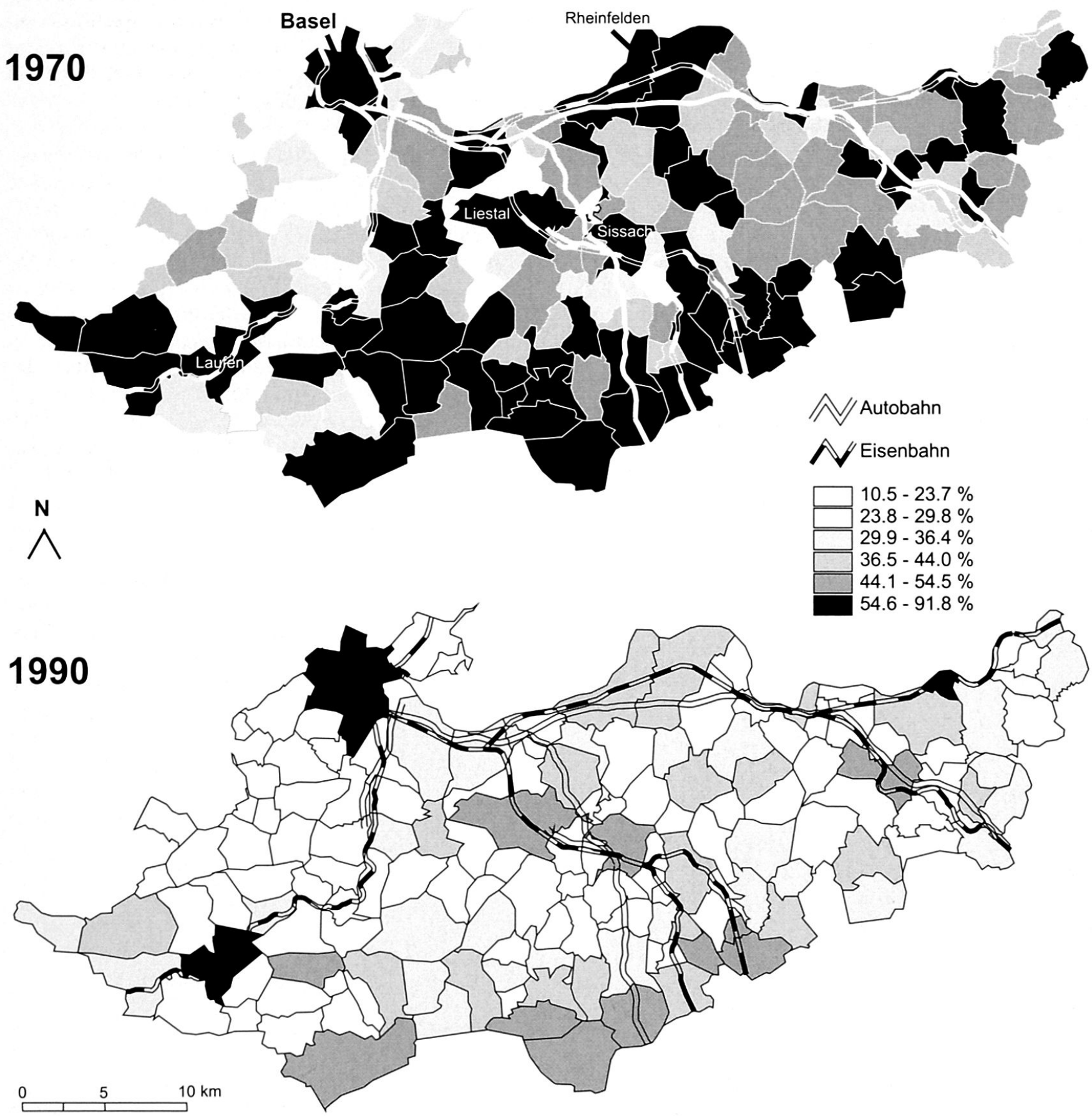

Karten 1 und 2: Prozentuale Anteile der innerhalb der Wohngemeinde arbeitenden Erwerbstätigen Percentage of employed persons within residential communities Pourcentages des actifs travaillant dans leur commune de domicile Quelle: Bundesamt Für Statistik: Volkszählungen 1970, 1980, 1990, eigene Berechnungen; Kartengrundlage: BFS GEOSTAT/L+T; Kartographie und Bearbeitung: P. REY 
70er Jahre verstärkte sich die bereits 1970 herrschende Dominanz des MIV bis 1980 signifikant. Gleichzeitig sank der ÖV-Anteil, während alle anderen Verkehrsmittel noch sehr viel stärker als der ÖV an Bedeutung verloren (Abb. 3).

Trotz der Entwicklungsumkehr in den 80er Jahren gilt es festzuhalten, dass auch 1990 dem MIV sowohl absolut als auch relativ die grösste Bedeutung innerhalb der Nordwestschweiz zukommt.

\section{Stadt Basel}

Aufgrund des grossen Zupendlerüberschusses beschränken sich die Analysen zur Stadt Basel auf die Entwicklung der Verkehrsmittelwahl der Zupendler. Die generelle Entwicklungsumkehr der 80er Jahre bildet sich auch in der Verkehrsmittelwahl der nach Basel pendelnden Erwerbstätigen ab (Abb. 4). Nachdem in den 70er Jahren sowohl der Anteil der MIVals auch der ÖV-Pendler leicht zunahm, worin sich der Bedeutungsverlust aller anderen Verkehrsmittel zeigt, stieg der Anteil der ÖV-Zupendler zwischen 1980 und 1990 von rund $43 \%$ auf $58 \%$. Gleichzeitig sank der prozentuale MIV-Zupendleranteil von $48 \%$ auf $36 \%$. Dieses Resultat lässt sich nur durch eine Betrachtung der Wohngemeinden der Zupendler erklären. Die Analysen zeigen, dass vor allem im Agglomerationsgürtel die Zahl der ÖV-Zupendler zwischen 1980 und 1990 stark zugenommen hat, während die Zahl der MIVZupendler zurückging. Dasselbe gilt für die in Subzentren wohnenden Zupendler der Stadt Basel. Obwohl die Bedeutung des öffentlichen Verkehrs auch für die aus den übrigen Gemeinden der Nordwestschweiz stammenden Zupendler in den 80er Jahren zugenommen hat, stieg hier die absolute Zahl der MIV-Zupendler auch in den 80er Jahren weiter an. Dies ist vor allem darum bedeutsam, weil der Anteil der von ausserhalb der Agglomerationsgemeinden stammenden Zupendler Basels in den 80er Jahren anstieg. Sollte sich die Suburbanisierung der Stadt Basel weiter verstärken, dann muss damit gerechnet werden, dass der Anteil der MIV-Zupendler wieder ansteigt, sollte es nicht gelingen, die Attraktivität des ÖV für die steigende Zahl der ausserhalb der Agglomerationsgemeinden wohnhaften Zupendler Basels zu steigern.

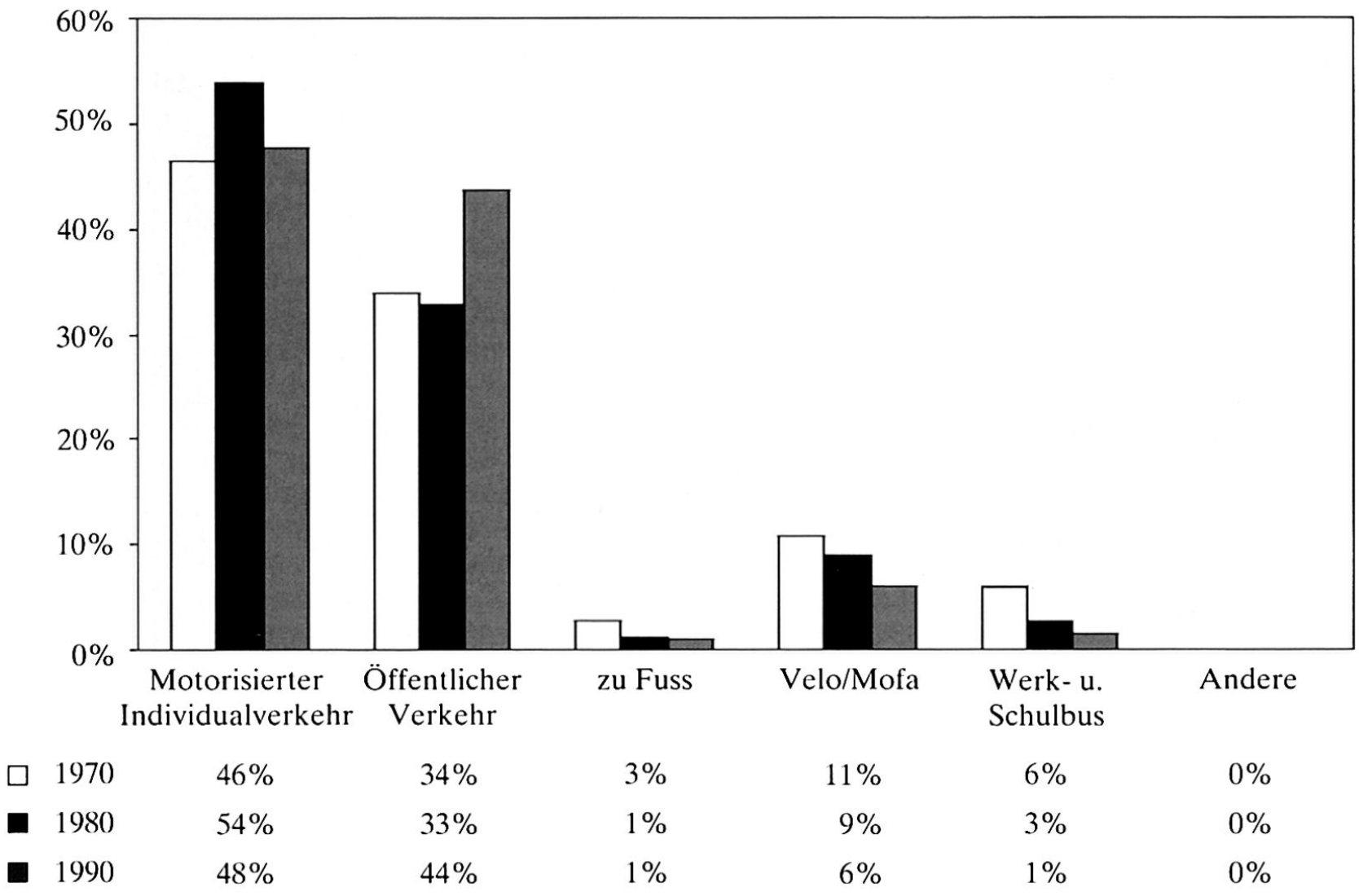

Abb. 3: Entwicklung der Verkehrsmittelwahl im interkommunalen Pendlerverkehr Choice of transport modes in intercommunal commuter traffic

Evolution du choix des moyens de transport dans le trafic pendulaire intercommunal Quelle: Bundesamt Für STATISTIK: Volkszählungen 1970, 1980, 1990 

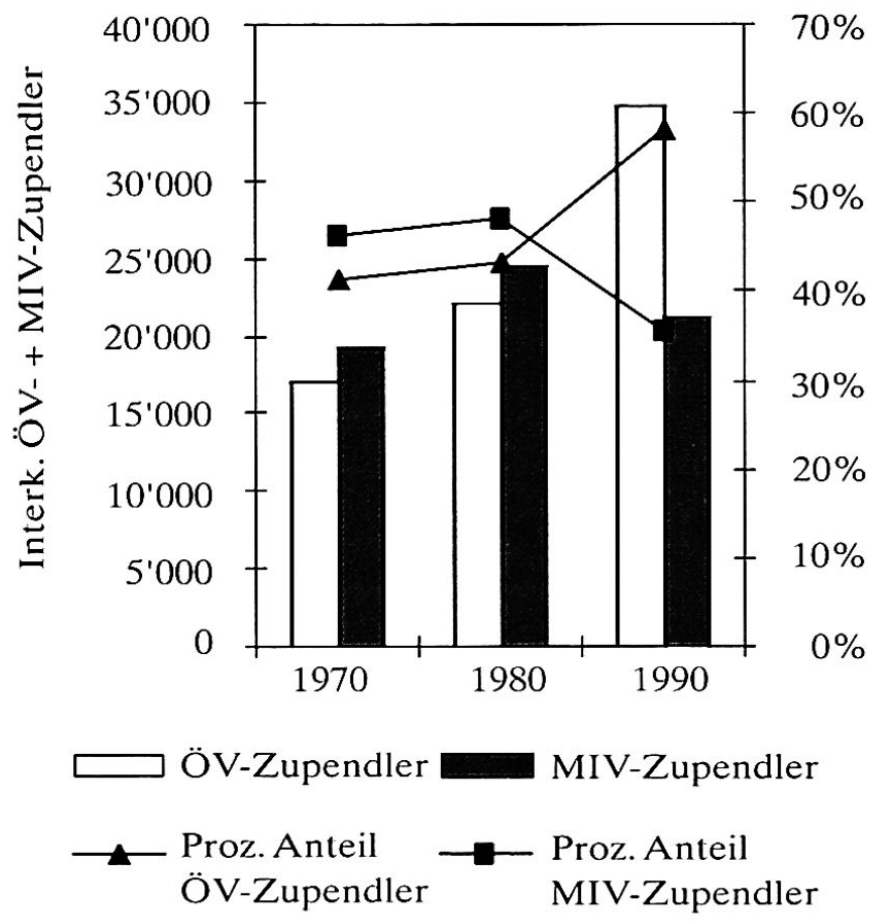

Abb. 4. Interkommunale Zupendler in die Stadt Basel (ÖV und MIV)

Intercommunal in-commuters to the City of Basel (public transport and motorised individual transport)

Pendulaires intercommunaux dans la ville de Bâle (transports publics et transports individuels motorisés) Quelle: Bundesamt Für StaTISTIK: Volkszählungen $1970,1980,1990$

\section{Interkommunaler Pendlerverkehr in den übrigen Agglomerationsgemeinden}

Die Dominanz der Wegpendler ist für die Agglomerationsgemeinden charakteristisch. Zudem dominiert im interkommunalen Zupendlerverkehr der MIV. Der Anteil der ÖV-Zupendler stieg wohl zwischen 1970 und 1990 von rund $30 \%$ auf $36 \%$. Aber auch 1990 benutzte noch mehr als jeder zweite Zupendler ein Verkehrsmittel des MIV (Personenwagen, Motorrad oder Roller). Der hohe MIV-Anteil im Zupendlerverkehr erklärt sich primär durch die grosse Zahl von MIV-Pendlern im interkommunalen «Agglomerationsbinnenverkehr»: Rund 57\% aller in einer Agglomerationsgemeinde wohnenden Erwerbstätigen, welche in einer anderen Agglomerationsgemeinde arbeiten, benutzten im Jahr 1990 ein MIV-Verkehrsmittel für den Arbeitsweg. Darin widerspiegelt sich die Schwäche der radial auf die Stadt Basel ausgerichteten Linien des öffentlichen Verkehrs: der ÖV kann den MIV auf Grund der Zeitverluste bei tangentialen Verbindungen nicht konkurrenzieren.
Entwicklung des interkommunalen Pendlerverkehrs in den übrigen Gemeinden der Nordwestschweiz

Während die Attraktivität des ÖV für den Pendlerverkehr zwischen der Stadt Basel und den Agglomerationsgemeinden in den 80er Jahren signifikant gesteigert werden konnte, zeigt sich bei der Betrachtung der interkommunalen Pendlermobilität in den übrigen Gemeinden der Nordwestschweiz ein anderes Bild: Der durchschnittliche Anteil der ÖV-Wegpendler sank in den 70 er Jahren auf rund $22 \%$. Trotz eines Anstiegs in den 80er Jahren betrug er auch im Jahr 1990 nur $32 \%$. Es kommt hinzu, dass der Anteil der MIV-Wegpendler in den 70 er Jahren von $45 \%$ auf $62 \%$ anstieg. In der leichten Abnahme bis 1990 auf $60 \%$ zeigt sich zudem, dass der Bedeutungsgewinn des ÖV in den übrigen Gemeinden der Nordwestschweiz primär nicht auf Kosten des MIV ging, sondern der ÖV vielmehr die alternativen Verkehrsmittel «Velo/Mofa» und «zu Fuss» konkurrenzierte.

\section{Verkehrsmittelwahl im intrakommunalen Pendlerverkehr}

Im Jahr 1990 pendelten neben 144'000 interkommunalen Pendler rund 100'000 Erwerbstätige innerhalb ihrer Wohngemeinde zwischen Wohn- und Arbeitsort. Die Analyse der Entwicklung der intrakommunalen Verkehrsmittelwahl wurde um die Verkehrsmittel «Velo/Mofa» und «zu Fuss» ergänzt, da diesen im intrakommunalen Pendlerverkehr eine nicht zu vernachlässigende Bedeutung zukommt (Abb.5).

\section{Stadt Basel}

Die Entwicklung des Anteils der intrakommunalen ÖVPendler zeigte, dass der ÖV für in der Stadt wohnende und arbeitende Erwerbstätige zwischen 1970 und 1990 zusätzlich an Bedeutung gewonnen hat (1970: 38\%, 1990: 57\%). Die starke Zunahme des ÖV im intrakommunalen Pendlerverkehr in Basel ging dabei auf Kosten der zu Fuss und motorisiert pendelnden Erwerbstätigen. Während der Anteil der MIV-Pendler zwischen 1970 und 1990 von $22 \%$ auf $14 \%$ sank, nahm der Anteil der zu Fuss pendelnden intrakommunalen Pendler zwischen 1970 und 1990 gar von $25 \%$ auf $14 \%$ ab.

Demgegenüber fiel in den übrigen Agglomerationsgemeinden die durchschnittlich höhere Bedeutung der zu Fuss, mit dem Velo/Mofa und dem MIV pendelnden Erwerbstätigen auf. Der auf den ersten Blick auffallende Unterschied in der intrakommunalen Verkehrsmittelwahl ist primär auf das kleinräumig gute Angebot des ÖV in der Stadt Basel zurückzuführen.

\section{Agglomerationsgemeinden (ohne die Stadt Basel)}

Trotz der Zunahme von rund 6\% seit 1970 pendelten auch 1990 in den Agglomerationsgemeinden durchschnittlich nur rund $13 \%$ aller intrakommunalen Pend- 


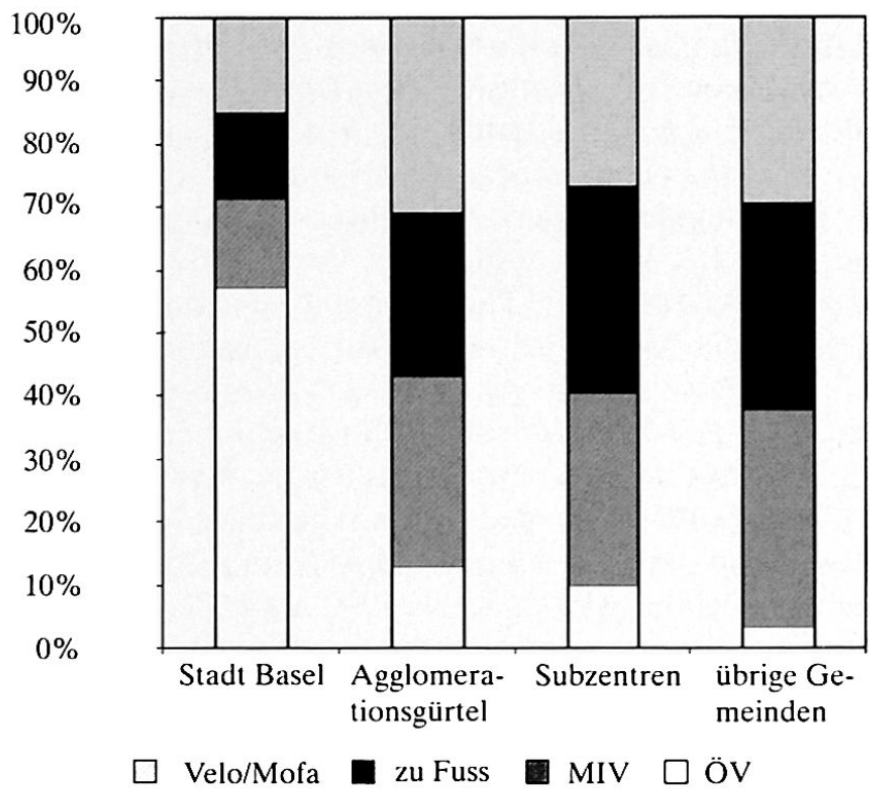

Abb. 5: Verkehrsmittelwahl der intrakommunalen Pendler 1990

Choice of transport by intracommunal commuters 1990

Choix du moyen de transport des pendulaires intracommunaux en 1990

Quelle: Bundesamt für StatıSTIK: Volkszählungen $1970,1980,1990$

ler mit dem öffentlichen Verkehr - verglichen mit 57\% in der Stadt Basel. Andererseits spielte sich das Wachstum der Velo/Mofa-Pendler auf einem im Vergleich zur Stadt Basel höheren Niveau ab. Während 1990 innerhalb der Stadt Basel nur rund 15\% der intrakommunalen Pendler mit dem Velo bzw. mit dem Mofa zwischen Wohn- und Arbeitsort pendelten, stieg der durchschnittliche Anteil in den Agglomerationsgemeinden zwischen 1970 und 1990 von $20 \%$ auf $30 \%$. Äquivalent zur Entwicklung in der Stadt Basel nahm auch im Agglomerationsgürtel die Zahl der zu Fuss pendelnden intrakommunalen Pendler ab. Der prozentuale Anteil an allen intrakommunalen Pendlern sank zwischen 1970 und 1990 von $42 \%$ auf $26 \%$. Damit pendelte auch 1990 - trotz der starken Abnahme - immerhin noch rund jeder vierte intrakommunale Pendler zu Fuss zwischen Wohn- und Arbeitsort, im Vergleich zu $14 \%$ in der Stadt Basel. Und trotz einer leichten relativen Abnahme bleibt die Bedeutung des MIV für den intrakommunalen Pendlerverkehr in den Agglomerationsgemeinden hoch. Rund durchschnittlich jeder dritte intrakommunal pendelnde Erwerbstätige benutzte 1990 noch das Auto bzw. ein Motorrad/Roller für den innerhalb der Wohngemeinde liegenden Arbeitsweg.
Subzentren und übrige Gemeinden der Nordwestschweiz Mit Ausnahme des öffentlichen Verkehrs zeigt sich in den Subzentren und den übrigen Gemeinden der Nordwestschweiz ein vergleichbares Bild der intrakommunalen Pendlermobilität. Auffällig auch hier ist die abnehmende Bedeutung der zu Fuss pendelnden intrakommunalen Pendler. Parallel dazu stieg sowohl der Anteil der intrakommunalen MIV-Pendler als auch der Velo/Mofa-Pendler. In den regionalen Subzentren legten im Jahr 1990 durchschnittlich 26\% der Erwerbstätigen den Arbeitsweg mit dem Velo bzw. mit dem Mofa zurück (1970: 18\%), während der Anteil in den übrigen Gemeinden der Nordwestschweiz zwischen 1970 und 1990 durchschnittlich von $18 \%$ auf $28 \%$ anstieg. Damit lag der Anteil der intrakommunalen Velo/Mofa-Pendler rund doppelt so hoch wie in der Stadt Basel. Gleichzeitig zeigt sich die zwischen 1970 und 1990 zunehmende Bedeutung des MIV. Während der Anteil der intrakommunalen MIV-Pendler in den Subzentren zwischen 1970 und 1980 von $23 \%$ auf $32 \%$ anstieg und bis 1990 leicht auf $30 \%$ fiel, stieg der Anteil in den übrigen Gemeinden der Nordwestschweiz zwischen 1970 und 1980 durchschnittlich von $21 \%$ auf $31 \%$. Andererseits setzte sich das Wachstum auch in den 80er Jahren - allerdings abgeschwächt - fort. Und während der öffentliche Verkehr in den Subzentren durchschnittlich zumindest den prozentualen Anteil von rund $2 \%$ auf $10 \%$ steigern konnte, blieb der ÖV für die intrakommunalen Pendler in den übrigen Gemeinden der Nordwestschweiz mit einem prozentualen Anteil von durchschnittlich 3\% auch im Jahr 1990 bedeutungslos.

Zusammenfassend kann gesagt werden, dass sich in den Entwicklungen der intrakommunalen Verkehrsmittelwahl die Bedeutung des öffentlichen Verkehrs als Funktion der städtischen Dichte zeigt. Während in der Stadt Basel im Jahr 1990 57\% aller intrakommunalen Pendler ein öffentliches Verkehrsmittel benutzten, sinkt der Anteil in den ländlich-peripheren Gemeinden auf $3 \%$. In den Gemeinden ohne nennenswerten öffentlichen Verkehr nahm der prozentuale Anteil der intrakommunalen MIV-Pendler auf Kosten der zu Fuss pendelnden Erwerbstätigen zu, während in der mit öffentlichem Verkehr gut erschlossenen Stadt Basel der ÖV das Pendeln zu Fuss substituierte.

\section{Diskussion der Ergebnisse}

Die Tatsache, dass im Jahr 1990 noch $40 \%$ der in der Stadt Basel wohnenden und in einer der Agglomerationsgemeinden arbeitenden Erwerbstätigen bzw. jeder dritte in die Stadt Basel pendelnde Erwerbstätige, der in einer der übrigen Agglomerationsgemeinden wohnte, mit den Verkehrsmitteln des MIV zwischen Wohn- und Arbeitsort pendelte, zeigt, dass trotz des dichten Netzes des ÖV und der Erfolge des Tarifverbundes weitere Fortschritte zur Attraktivitätssteigerung möglich sind. 
Dadurch liesse sich die Verkehrsbelastung in der Agglomeration Basel durch den MIV reduzieren. Dabei darf aber nicht vergessen werden, dass es sich bei der Beeinflussung der Verkehrsmittelwahl zu Gunsten des öffentlichen Verkehrs immer nur um eine «Zweitbestlösung» handeln kann. Und bei der subventionierten Verbilligung des ÖV handelt es sich eigentlich um eine «Drittbestlösung», denn durch die Externalisierung von Verkehrskosten auf die Allgemeinheit (Finanzierung über Steuermittel) geht vergessen, dass Mobilität - unabhängig vom Verkehrsmittel - zu billig ist. Die «Zweitbestlösung» bestünde daher darin, den MIV zu verteuern und durch restriktive Massnahmen an den Zielorten, wie z.B. Road-Pricing in der Agglomeration, Parkplatzbeschränkungen in Wohngebieten und eine Ausweitung der autofreien Zonen, einzuschränken. Dies würde die Attraktivität des ÖV gegenüber dem MIV erhöhen, ohne dass durch Subventionierung des ÖV die Staatskassen weiter belastet würden.

Die beste aller Lösungen bestünde darin, dafür zu sorgen, dass der Entwicklungstrend der zunehmenden Trennung von Wohnen und Arbeiten gebrochen werden kann. Dies wiederum kann nur dann geschehen, wenn es gelingt, die Wurzeln der suburbanen Utopie freizulegen, d.h. die Frage zu beantworten, was viele Erwerbstätige (inkl. Angehörige) dazu veranlasst, Beschäftigungszentren wie die Stadt Basel in Richtung «grüner Wiese» zu verlassen, und dabei das tägliche Pendeln zwischen Wohn- und Arbeitsort in Kauf zu nehmen. Erste Anstrengungen - z.B. in Form einer «Wanderungsbefragung» (STATISTISCHES AMT DES Kantons BASEL-STADT 1999) - wurden bereits unternommen. Es bedarf aber weiterer Bemühungen, die Motive und Präferenzen für die Wohnstandortwahl zu bestimmen, um dadurch bessere Rahmenbedingungen für das Wohnen schaffen zu können.

Zudem bedürfen insbesondere folgende Instrumente einer stärkeren Gewichtung:

- Ausgleich von Zentrumslasten (spillovers): Da das kernstädtische Angebot an öffentlichen Leistungen auch den Einwohnerinnen und Einwohnern der übrigen Nordwestschweiz zugute kommt, sollte der horizontale und vertikale Lastenausgleich ausgebaut werden, so dass Entscheidungsträger (= Stadt Basel), Kostenträger (= Stadt Basel) und Nutzniesser (= Regionsbevölkerung) räumlich übereinstimmen (= Schaffung fiskalischer Äquivalenz).

- Ausrichtung des staatlichen Handelns am Konzept des Standortmarketings: Standortmarketing ist ein marktorientiertes Führungs- und Managementkonzept zur Entwicklung von Städten und Regionen. Gefordert wird die systematische Ausrichtung aller immobilen Standortfaktoren wie Infrastruktur, gesetzliche Rahmenbedingungen, Steuersystem usw. auf die Bedürfnisse definierter «Kundengruppen». Es geht folglich nicht mehr nur um die sektorale Optimierung von öffentlichen Dienstleistungen, wie dies heute oftmals noch der Fall ist. Vielmehr steht die Definition von Kunden (Bevölkerungsgruppen, Branchen, regionale Schlüsselindustrien) und die Ausrichtung aller immobilen Produktionsfaktoren auf die Bedürfnisse dieser Kundengruppen im Zentrum.

Als besonders bedeutender städtischer Mangel erscheint die Umweltsituation und das Wohnumfeld. Gerade darin zeigt sich ein mit der Bevölkerungssuburbanisierung und der zunehmenden interkommunalen Pendlermobilität verbundenes Dilemma der Kernstadt: Dadurch dass die Arbeitsplätze städtisch konzentriert bleiben, während sich Erwerbstätige und Angehörige nach Suburbia verabschieden, erhöht sich der Pendlerverkehr, wobei vor allem der steigende MIV die Umweltsituation und das Wohnumfeld in der Stadt weiter verschlechtert, so dass ein sich selbstverstärkender Prozess in Gang gekommen ist. Gleichzeitig bleibt die Stadt Basel aber auch das kulturelle Zentrum und das Einkaufszentrum der in das Umland wegziehenden Bevölkerung, so dass es wenig erstaunt, dass kernstädtische Anstrengungen einer Verbesserung der Lebensqualität, der Umweltsituation und des Wohnumfeldes durch eine Einschränkung des motorisierten Individualverkehrs bei der suburbanen Bevölkerung und dem städtischen Gewerbe auf mangelnde Akzeptanz stossen.

Es wird das Ziel einer an der nachhaltigen Gesamtentwicklung interessierten Politik sein müssen, das in vielerlei Hinsicht unbewältigte Problem eines ungebrochenen Mobilitätswachstums zu lösen. Denn so erfolgreich das im Verhältnis zum MIV stärkere personenbezogene Wachstum des ÖV im Pendlerverkehr auch erscheint, das Resultat der bisherigen Anstrengungen ist nur aber immerhin - eine Verschiebung des Modal Splits. Die Verkehrsmittelwahl konnte durch den Einsatz von beträchtlichen finanziellen Mitteln zu Gunsten des ÖV beeinflusst werden. Dabei muss einschränkend der personenbezogene Gehalt der Analysen betont werden, da aufgrund fehlender Pendlerdistanzen keine statistisch gesicherten Aussagen zur Entwicklung der durch den MIV verursachten Umweltbelastung möglich sind. Doch trotz der ökonomischen Quantifizierung und Monetarisierung eines Teils der effektiven gesellschaftlichen Mobilitätskosten (Gebäudeschäden, Lärm- und Unfallkosten usw.) harrt die Kostenwahrheit weiterhin ihrer politischen Verwirklichung.

\section{Literatur}

Bundesamt für Statistik (1997): Volkszählung 1990. Pendlermobilität in der Schweiz. - Bern.

ECONCEPT (1998): Kosten der Luftverschmutzung in den Kantonen Basel-Stadt und Basel-Landschaft. - Schlussbericht i. A. des Lufthygieneamtes beider Basel, Liestal.

FrEY, R. L. (1996): Stadt: Lebens- und Wirtschafts- 
raum. Eine ökonomische Analyse. - Zürich: vdf Hochschulverlag.

Rey, P. (1999): Pendlermobilität in der Nordwestschweiz. Entwicklung der interkommunalen Pendlerbeziehungen und der Verkehrsmittelwahl zwischen 1970 und 1990. - Lizentiatsarbeit am Geographischen Institut der Universität Basel,Abteilung Humangeographie/ Stadt- und Regionalforschung.

Schneider-Sliwa, R., Kampschulte, A., Nommel, J.-U., Sandtner, M., Strassmann, R. \& CH. WaffenschmidT (1999): Bevölkerungsstruktur und Bevölkerungsdynamik beider Basel. - = Reihe Stadt und Region 1, Basel. Statistisches Amt des Kantons Basel-Stadt (1999): Wanderungsbefragung 1998.

\section{Zusammenfassung: Pendlermobilität in der Nord- westschweiz - Entwicklung der Pendlerbeziehungen und der Verkehrsmittelwahl zwischen 1970 und 1990}

Im Kontext der funktionalen Entmischung wurde das Wachstum des Pendlerverkehrs am Beispiel der Region Nordwestschweiz thematisiert. Es bestätigte sich, dass die interkommunale Pendlermobilität stark zugenommen hat. Der Anteil der interkommunalen Pendler stieg zwischen 1970 und 1990 von 33\% auf $52 \%$. Trotz der Bevölkerungssuburbanisierung dominiert der interkommunale Pendlerverkehr innerhalb und in die Agglomeration Basel, da sich das Arbeitsplatzangebot weiterhin in der Agglomeration Basel konzentriert. Im interkommunalen Pendlerverkehr ist in den 80er Jahren der öffentliche Verkehr stärker gewachsen als der motorisierte Indidividualverkehr. Dies im Unterschied zum starken Wachstum des MIV in den 70er Jahren. In der Stadt Basel benutzten 1990 $57 \%$ aller intrakommunalen Pendler ein öffentliches Verkehrsmittel. Dieser Anteil betrug 1990 in den ländlich-peripheren Gemeinden durchschnittlich 3\%. Um dem Trend der zunehmenden Trennung zwischen Wohnen und Arbeiten entgegenzuwirken, sind einerseits Massnahmen zur Steigerung der Wohnstandortqualität in den Zentrumsgemeinden zu ergreifen. Andererseits kann längerfristig die Zunahme des Pendlerverkehrs im allgemeinen und des motorisierten Individualverkehrs im speziellen nur durch Kostenwahrheit im Verkehr (Road-Pricing usw.) und durch restriktive Massnahmen in den vom Verkehr betroffenen Gemeinden verhindert werden.

\section{Summary: Commuter Mobility in North-West Swit- zerland - Changes in traffic and choice of transport mode between 1970 and 1990}

This paper analyzes the growth of cummuting in NorthWest Switzerland. A strong increase in intercommunal commuting could be confirmed. In 1990, intercommunal commuting accounted for $52 \%$ of total commuting (33\% in 1970). Despite suburbanisation, intercommunal commuting continues to focus on the city centre and the agglomeration of Basel due to the availability of jobs there. Whereas the 1970s saw a strong increase of motorised individual transport for commuting purposes, by the 1980s public transport had the upper hand. Within Basel, $57 \%$ of all intracommunal commuters made use of public transport in 1990. In contrast, the corresponding figure in the rural-peripheral communities was on average $3 \%$. In order to break the trend towards division of work and living, measures need to be implemented to improve the quality of living in the core communes. The growth of both commuting by public and motorized individual transport can only be combated in the longterm by accounting for the true-cost of transport (road-pricing etc.), and with restrictive measures within communities negatively affected by traffic.

\section{Résumé: Mobilité pendulaire dans le nord-ouest de la Suisse - Evolution de la pendularité et du choix du moyen de transport entre 1970 et 1990}

Le nord-ouest de la Suisse a été choisi comme exemple pour étudier l'accroissement du trafic pendulaire dans le contexte de la dissociation fonctionnelle. Cette étude a confirmé la forte augmentation de la mobilité pendulaire intercommunale. Entre 1970 et 1990, le pourcentage des pendulaires intercommunaux est passé de $33 \%$ à $52 \%$. Malgré la suburbanisation de la population, le trafic pendulaire intercommunal domine à l'intérieur de l'agglomération bâloise et en direction de celle-ci, puisque l'offre en places de travail se concentre encore dans cette agglomération. Dans le trafic pendulaire intercommunal, les transports publics ont connu dans les années quatre-vingt une plus forte croissance que les transports individuels motorisés (ces derniers étaient par contre ceux qui s'étaient le mieux développés dans les années septante). Dans la ville de Bâle, $57 \%$ de l'ensemble des pendulaires intracommunaux ont utilisé en 1990 un moyen de transport public. La même année, cette part était en moyenne de 3\% dans les communes périphériques rurales. Pour agir contre la dissociation qui tend à croître entre domicile et travail, il s'agit d'une part de prendre des mesures pour élever la qualité du lieu de résidence dans les communes centrales. D'autre part, à plus long terme, seules la vérité des coûts dans les transports (Roadpricing, etc.) et des mesures restrictives dans les communes concernées par le trafic permettront de freiner l'augmentation du trafic pendulaire en général et des transports individuels motorisés en particulier.

Lic. phil. Patrick Rey, St. Galler-Ring 206, CH-4054 Basel.

Manuskripteingang/received/rentrée du mansucrit: 14.12.2000

Annahme zum Druck/Accepted for publication/ acceptation à l'impression: 21.6.2001 